\section{Telehealth and the COVID-19 Pandemic: International Perspectives and a Health Systems Framework for Telehealth Implementation to Support Critical Response}

Arindam Basu', Craig Kuziemsky², Magdala de Araújo Novaes ${ }^{3}$, Araujo Kleber ${ }^{4}$, Fernando Sales ${ }^{5}$, Najeeb Al-Shorbaji ${ }^{6}$, José F. Flórez-Arango ${ }^{7}$, Shashi B. Gogia ${ }^{8}$, Kendall $\mathrm{Ho}^{9}$, Inga Hunter $^{10}$, Sriram lyengar ${ }^{11}$, Oommen John ${ }^{12}$, Sheila John ${ }^{13}$, Gumindu Kulatunga ${ }^{14}$, 15 , Vije Kumar Rajput ${ }^{16}$, Prasad Ranatunga ${ }^{14}$, 15 , Jai Ganesh Udayasankaran ${ }^{17}$

1 University of Canterbury, Christchurch, New Zealand

2 MacEwan University, Edmonton, Canada

3 Medical Sciences Center, Telehealth Center, Clinics Hospital, Federal University of Pernambuco, Recife, Brazil

4 Telehealth Center (NUTES), Federal University of Pernambuco, Recife, Brazil

${ }^{5}$ Technology and Geosciences Center, Telehealth Center, Federal University of Pernambuco, Recife, Brazil

${ }^{6}$ eHealth Development Association of Jordan, Amman, Jordan

7 Texas A \& M University, College Station, United States of America

8 Society for Administration of Telemedicine and Healthcare Informatics, New Delhi, India

9 University of British Columbia, Vancouver, Canada

10 Massey University, Palmerston North, New Zealand

${ }^{11}$ University of Arizona, Phoenix, United States of America

${ }^{12}$ George Institute for Global Health, University of New South Wales, Sydney, Australia

${ }^{13}$ Sankara Nethralaya, Chennai, India

${ }^{14}$ Postgraduate Institute of Medicine, University of Colombo, Colombo, Sri Lanka

15 University of Southampton, Southampton, UK

${ }^{16}$ Keele University, Staffordshire, UK

17 Sri Sathya Sai Central Trust, Puttaparthi, India

\section{Summary}

Objectives: Telehealth implementation is a complex systems-based endeavour. This paper compares telehealth responses to (COrona Vlrus Disease 2019) COVID-19 across ten countries to identify lessons learned about the complexity of telehealth during critical response such as in response to a global pandemic. Our overall objective is to develop a health systems-based framework for telehealth implementation to support critical response.

Methods: We sought responses from the members of the International Medical Informatics Association (IMIA) Telehealth Working Group (WG) on their practices and perception of telehealth practices during the times of COVID-19 pandemic in their respective countries. We then analysed their responses to identify six emerging themes that we mapped to the World Health Organization (WHO) model of health systems.

Results: Our analysis identified six emergent themes. (1) Government, legal or regulatory aspects of telehealth; (2) Increase in telehealth capacity and delivery; (3) Regulated and unregulated telehealth; (4) Changes in the uptake and perception of telemedicine; (5) Public engagement in telehealth responses to COVID-19; and (6) Implications for training and education. We discuss these themes and then use them to develop a systems framework for telehealth support in critical response. Conclusion: COVID-19 has introduced new challenges for telehealth support in times of critical response. Our themes and systems framework extend the WHO systems model and highlight that telemedicine usage in response to the COVID-19 pandemic is complex and multidimensional. Our systems-based framework provides guidance for telehealth implementation as part of health systems response to a global pandemic such as COVID-19.

Keywords

Telemedicine, telehealth, COVID-19, coronavirus, pandemic, health systems

Yearb Med Inform 2021:126-33

http://dx.doi.org/10.1055/s-0041-1726484 


\section{Introduction}

The COrona VIrus Disease 2019 (COVID-19) pandemic brought a transformative shift in medical care and public health delivery. When the COVID-19 pandemic began to spread worldwide in early 2020, its infectiousness and rapid transmission required countries to look for digital means to deliver healthcare services that would provide necessary care for patients while also supporting physical distancing and other public health restrictions. As a result, traditional face-to-face clinical encounters needed to be complemented with digital solutions, resulting in the adoption of virtual care tools such as telehealth to became commonplace worldwide $[1,2]$. While telehealth and other virtual care tools existed pre-COVID, the need for public health practices such as physical distancing and a desire to keep people out of high-risk settings such as hospitals accelerated the adoption of telehealth. The challenges of the COVID-19 pandemic and disruption on health service delivery due to lockdowns or other public health measures resulted in a rapid implementation and scaling up of digital health innovations. These solutions focused on planning, response and management of COVID-19 including disease surveillance, patient screening, contact tracing, patient diagnosis and follow-up, supply chain management, staff training, and research such as predictive modelling of COVID-19 spread [3-5]. As part of this digital health response to COVID-19, many countries have introduced new regulatory frameworks, or revised existing frameworks to allow telemedicine to play a vital role in the societal response to COVID-19 [2, 6]. The pandemic has shown us what is possible for telehealth delivery and there is global interest to sustain the COVID-19 facilitated increase in telehealth usage after the pandemic $[7,8]$.

Despite the potential of telehealth for health professionals and health delivery systems to extend care delivery beyond routine care such as face-to-face delivery, implementing telehealth presents several challenges including scale up and long-term sustainability [9]. Telehealth implementation is a sociotechnical endeavour and human-centred design approaches, and local care delivery practices and enablers must be considered [10]. Implementing new technologies during a global pandemic adds to the existing complexities around change management, patient engagement, scale up, prioritization of care and ensuring equity of services provided. Unintended consequences are a common occurrence post health information technology (HIT) implementation. Various unintended consequences (UIC) including community, patient safety, information retrieval, individual-collaborative interchanges, and workflow issues have been described [6, 11-14]. UICs remind us that HIT implementation is not without risk and expedited implementation of HIT such as telehealth in response to the COVID-19 pandemic could present an increased risk of UICs. However, at the same time we want to use COVID-19 as an opportunity to learn how we might increase our capacity for telehealth delivery while also accounting for UICs such as privacy and security, ethical and legal concerns, and social issues [10,15]. Regardless of whether HIT adoption occurs over years or weeks such as during COVID-19, it is still a complex adoption process and we must account for implementation factors such as workflow, organizational, social, and ethical and legal concerns.

A high performing health system is one that accounts for all aspects of a health system and may be more resilient in response to COVID-19 [16]. The World Health Organization (WHO) model of health systems includes six building blocks (service delivery, health workforce, medical products, vaccines and technology, financing and governance/leadership) and four system goals/ outcomes (improved health, responsiveness, social and financial risk protection and improved efficiency) $[17,18]$. The system building blocks and outcomes combined with systems thinking approaches enables the design of interventions such as policy or health information technology (HIT) to support health system objectives [19, 20]. The focus of our working group is on medical informatics and more specifically telehealth, but we recognize that HIT is only one component of a health system that must be aligned with other system components.
Telehealth is a classic example of a health system in that that it consists of several interrelated parts that must work in unison for telehealth to effectively contribute to healthcare delivery [21].

While COVID-19 has identified many opportunities for telehealth, it has also identified challenges that the medical informatics community must overcome as part of developing a sustainable telehealth system for supporting critical response such as a global pandemic [1,22]. Incorporating systems thinking into how we conceptualize telehealth implementation would help us leveraging the strengths of telehealth while addressing the system-based challenges of using telehealth during critical response. To date, there is no systems-based framework for telehealth delivery. We suggest that a system-based framework could allow us to understand how telehealth must evolve or be adapted for critical response such as the COVID-19 pandemic.

In this paper we describe the international experiences in seven countries of implementing telemedicine/ telehealth in the context of the ongoing COVID-19 pandemic. We then use the international perspectives to develop a health systems-based framework for telehealth implementation to support critical response.

\section{Materials and Methods}

All members of the Telehealth Working Group (WG) of the International Medical Informatics Association (IMIA) were invited to provide their inputs and reflections on telehealth use in response to the COVID-19 pandemic. The Telehealth WG consists of approximately 100 members worldwide including researchers, practitioners, and academics. The question posed to WG members was "Describe how telemedicine was used in your country in response to the COVID-19 pandemic, including pros and cons of telemedicine usage". We received data from the members of the IMIA working groups in ten countries: Australia, Brazil, Canada, Colombia, India, Jordan, New Zealand, Sri Lanka, The United States, and The United Kingdom. These countries represented 
different health system delivery models and perspectives and critical response to COVID-19, including variations in success in response to COVID-19. The complete text of the narratives is presented in the accompanying public GitHub repository [23].

Data was collected from WG members from September-November 2020. Starting in mid-October the authors did an ongoing content analysis of the data to identify emergent themes related to telehealth use during COVID-19. AB and CK led the content analysis with all authors contributing to it. Disagreements in the analysis were resolved by group consensus.

We used the WHO Health System model we described earlier, and our prior work research at comparing international telehealth implementation, to guide the analysis and to identify health system considerations for telemedicine use during critical response $[9,17,24,25]$. Our previous research and the WHO Health System model are complementary in that we identified specific issues related to telehealth implementation including different models of patient-centred connected health, the need to balance patient needs with technological capability, and emerging privacy needs that can arise from informal telehealth implementation, while the WHO model describes the broad concepts that comprise a health system.

\section{Results}

Our analysis identified six themes that describe telemedicine experiences during the COVID-19 pandemic and the lessons learned to implement telehealth to better support times of critical response such as COVID-19. The themes were: (1) government legal or regulatory aspects of telehealth; (2) increase in telehealth capacity and delivery; (3) regulated and unregulated telehealth; (4) changes in the uptake and perception of telemedicine; (5) public engagement in telehealth responses to COVID-19; and (6) implications for training and education.

In the sections below we describe and provide quotes to support each theme. The country where each text came from is enclosed within parentheses for reference.

\subsection{Government, Legal or} Regulatory Aspects of Telehealth

This theme describes government, legal or regulatory aspects that impacted countries abilities to ramp up telehealth delivery in response to the COVID-19 pandemic.

(India): "The Government of India issued an advisory for those with chronic conditions to avoid visits to healthcare facilities for non-emergency consultations, resulting in significant challenges to those with chronic conditions and multiple morbidities. These disruptions led to the accelerated release of the Telemedicine Practice Guidelines 2020 (TPG) which provides a legal framework for delivery of health care remotely through telemedicine services by the Board of Governors in supersession of the Medical Council of India which caters to practitioners of modern Medicine". (India): "The Integrated Disease Surveillance Program (IDSP), which is a nation-wide surveillance system for epidemic prone diseases has been activated by the Ministry of health and family welfare towards COVID response, and is being further strengthened with substantive digital inputs"

(Brazil): "Brazilian telemedicine law has been promulgated and it is valid as long as the pandemic takes place".

(Brazil): "Legal authorization of telemedicine by a Federal Law [April 15 ${ }^{\text {th }} 2020$ ] - Several practices have been permitted for remote consultations and prescriptions, complying to some requirements to provide security to the process and safety for the patients".

(Sri Lanka):" the Country did not have any regulations or guidelines specific to Telemedicine services when the pandemic started [26]. Health Information Unit of the Ministry of Health, Sri Lanka made a set of non-formal guidelines to assist voluntary participating doctors when using Telehealth services and trained them using online methods.. As for further development the National eHealth Guidelines and Standards (NeGS) are currently being updated to include Telehealth services"

(New Zealand):"... rapid introduction of electronic prescribing ... by the Ministry of Health, the Medical Council of New
Zealand and the NZ Telehealth Leadership Group, ... determined new rules for electronic prescribing using NZePS (NZ ePrescribing services) with a manual signature exempt temporary waiver from the NZ Government". "Expansion of a digital national contact tracing system". "The Medical Council made special provisions for the return for doctors who had retired with the last 3 years to be able to return to practice if needed".

(Colombia): "When COVID struck, Colombia issued an exception law, where all face to face and elective procedures were suspended ... law facilitated telemedicine and telehealth services that required direct interaction with patients"

(UK):" NHS England and NHS Improvement (NHSEI) provide a national direction on service improvement and transformation, governance and accountability, standards of best practice, and quality of data and information. NHSX (nhsx.nhs.uk) is responsible for driving digital transformation and leading policy implementation and change".

(Canada):" One benefit of COVID-19 was that the rapid shift to virtual care required adapting physician fee schedules, including new billing codes and updated practice guidelines. While each province and territory implemented their own billing code for virtual care, there were many similarities across Canada. By the end of March 2020, all provinces and territories had some version of a billing code to support virtual care".

(Australia):"The federal government has opened up billing codes in the national system to allow telephone or video consultations for virtually all types of consultations".

\subsection{Increase in Telehealth Capacity and Delivery}

This theme describes various ways in which countries increased telehealth capacity and delivery in response to the COVID-19 pandemic.

(India): "eSanjeevaniOPD, a free online national OPD service from Government of India has enabled patients to consult 
doctors from home. This real time telemedicine service facilitates safe and structured video-based consultations via web application as well as mobile app". (India): United Care Development Services (UCDS) an NGO in India has integrated cloud telephony service with a free health information systems application. This arrangement has facilitated a two-way clinical documentation exchange between the patient and the practitioner. The consultation summary and prescription conforming with the Government guidelines are prepared by the practitioners and sent to the patient via WhatsApp. These improvisations have led to better experience for the patients seeking phone-based consultations and professional satisfaction for the medical practitioners providing this free service.

(India): "In India, several health startups offered video consultations".

(Sri Lanka):" Even though COVID case rate was low in Sri Lanka, during first half of 2020, the general public was unable to visit a health care facility even if it was next door due to strict movement regulations. During the COVID pandemic in the first half of 2020, the general public was unable to visit a health care facility even if it was next door. Alternative methods were needed urgently for screening of suspected cases as well as for the follow-up of chronic diseases who need long term care. This vacuum resulted in wide expansion of Telehealth service providers".

(Colombia): "Analysing the National registry of providers we can see the explosion of new enabled registered services. There is a high concentration in "reference“ services".

(Canada):" the BC Ministry of Health, in association with a group of health organizations including the Rural Coordination Centre of British Columbia, First Nations Health Authority, BC Emergency Medicine Network, Doctors of BC, Provincial Health Services Authority, and Providence Healthcare, set up a network of virtual care to support clinicians-to-clinicians and clinicians-to-patients telehealth services, collectively named as Real Time Virtual Support (RTVS) services".
(UK) "Licences for online consultation software are purchased from a list of accredited suppliers, via a dynamic purchasing system".

(Brazil):" several initiatives conducted by local entities and the State Governments. The State of Sao Paulo has supported the TeleICU Project, evolving several hospitals to share practices, intensive care protocols and clinical trials related to COVID-19 pandemics".

(Jordan): Providing free transportation services to patients in emergency situation to reach to hospitals and providing hot line services. The government commissioned the development of mobile apps for contact racing, others for ePrescriptions especially for chronic diseases allowing for renewal of prescritions without having to visit physically the healthcare centre. Other applications were developed to allow for making appointments without having to come to the hospital.

\subsection{Regulated and Unregulated Telehealth}

This theme describes the different telehealth tools, both formal and informal, that were used during the COVID-19 pandemic.

(India): "Most teleconsultations are still largely through WhatsApp and Telephone with emails coming a distant $3^{\text {rd }}$."

(India): "Laws applicable to telemedicine in India are those governing practice of medicine and laws governing Information Technology transactions. Personal Data Protection Bill, 2019 pending approval from the Parliament is expected to further shape practice of telemedicine when it becomes an Act."

(Sri Lanka): "The MyHealth Sri Lanka" application was made to empower citizens with correct knowledge for correct decision making" and to collaborate between government sector and private sector [27]. (New Zealand):" The Ministry of Health ... introduced the NZ COVID Tracer App, a digital diary using QR codes scannable on mobile phones".

(United States):" AZCOVIDTXT is a powerful bilingual (English and Spanish) system to help the citizens of Arizona, especially the more vulnerable and underserved populations, successfully cope with this pandemic".

(UK):" A Digital First Primary Care approach supports patients to use online tools to access all primary care services, such as receiving advice, booking and cancelling appointments, having a consultation with a healthcare professional, receiving a referral and obtaining a prescription."

(UK):" Text messaging is used to communicate with patients for a range of reasons including booking appointments, self-help web links, attaching documents/ images and flu/covid-19 campaigns".

(Brazil): "Chatbot or with the Coronavirus SUS application. The action aims to reach 120 million Brazilians, helping the early identification of people with signs and symptoms of flu syndrome, vulnerable people and mapping areas of risk of contagion".

(Brazil): "a large use of WhatsApp and mobile applications (e.g., chatbots) is observed in Brazil for provider-provider and provider-patient communication, both in public and private healthcare service".

(Jordan): "The use of normal telephone lines, WhatsApp and SMS services increased as patients asked for assistance, advice and information. These services have never been regulated and there is no medicolegal accountability as a result."

\subsection{Change in the Perception or Uptake of Telehealth}

This theme looks at how the use of telehealth tools evolved because of the COVID-19 pandemic.

(India): "Its use for tele-consult was largely frowned upon but has been demand driven by patients as well as forced upon by the clinicians who are themselves vary of facing the patients directly. The concept of Telehealth has shifted from remote care to a "I would rather not see a patient physically"."

(India): "The Government of India has included telemedicine as one of the key 
building blocks proposed in the National Digital Health Mission (NDHM) and the National Health Authority who is the implementing agency of NDHM has invited Proof of Concept ( $\mathrm{PoC})$ for telemedicine building block."

(India): The Insurance Regulatory and Development Authority of India (IRDAI) has asked the insurers in the country to allow claim settlement for telemedicine consultation wherever normal consultation with a medical practitioner is allowed in the terms and conditions of the policy contract.

(Brazil): "Healthcare professionals, not only physicians, have embraced remote care and a lot of fear of using technology has been surpassed ... Self-care algorithms have been adopted as an acceptable resource of health care by patients, healthcare providers and policy makers".

(New Zealand): "Healthcare services thus had 48 hours to switch service delivery from in-person to telehealth, and this was over a weekend! GPs were asked to switch $70 \%$ of their consultations to telehealth ... Secondary care providers (hospital based care) started to run telehealth consultations in their outpatient departments". (UK):" In England general practice has moved form $90 \%$ of face to face consultations to $85 \%$ remote consultations mainly using telephone, video consultation or texting."

(Sri Lanka): Medical practitioners volunteered to provide Telehealth services coordinated through the "Myhealth Sri Lanka" application. Healthcare professionals as well as patients attitude on distant care methodology changed even though Sri Lankan COVID-19 case rates were low compared to other counties. Private sector was eager to collaborate with government sector in sharing telehealth platforms to provide health services for the citizens.

(Brazil):" Healthcare professionals, not only physicians, have embraced remote care and a lot of fear of using technology has been surpassed ... Self-care algorithms have been adopted as an acceptable resource of health care by patients, healthcare providers and policy makers".

\subsection{Public Engagement in Tele- health Responses to COVID-19}

This theme looks at public engagement opportunities that arose because of the COVID-19 pandemic.

(India): A volunteer based network of health focused innovators came together on a common technology platform as Project StepOne.

(India): The Telemedicine Society of India in partnership with the Digital Health India Association pooled a team of volunteer resource persons who designed an online "Train to Practice Telemedicine Certificate Course".

(India): "Telemedicine business models have been evolving from a patient-healthcare provider model (person to person), to an institutional level, including, but not limited to, hospitals, pharmacies, clinical analysis laboratory".

(Brazil): "NUTES-UFPE implemented a remote monitoring center for patients with flu-like symptoms [possible covid cases] and mental health issues related to pandemic context. This service was, on the first moment, composed by volunteers and after some weeks,".

(UK):" Volunteers have been engaged supporting over 2 million people at risk such as delivering food and medicines, driving patients to appointments and phone calls for the isolated. They are managed through a dedicated mobile app."

(Brazil):" Hundreds of volunteers have been trained for remote guidance and consultation. Gradually, other specialty agendas were opened, and the mental health agenda became the most demanded service to face mental suffering due to social and distance isolation".

(Jordan): "During COVID-19 especially in lockdown and curfew times, the only possible way to deliver health care services and information was via telehealth support. Patients did like this method as many of them did not really need to be at the hospital physically. They needed advice, information, follow up and/or confirmation or opinion of certain health status."

\subsection{Implications for Training and Education}

This theme looks forward as to what some of the lessons learned are from the COVID-19 pandemic implications for telehealth training and education.

(India): "Delivery of 'Train to Practice Telemedicine Certificate Course' began within ten days of the release of the telemedicine practice guidelines. This online course delivered as a webinar consisted of four modules of 20 - 30 minutes duration each was followed by questions and answers apart from pre-course and post-course quiz".

(India): "Ministry of Health \& Family Welfare, Govt. of India is implementing an eHealth project including Telemedicine on National Medical College Network (NMCN) for interlinking the Medical Colleges across the country with the purpose of e-Education and National Rural Telemedicine Network for e-Healthcare delivery".

(New Zealand): "Health Informatics New Zealand and the NZ Telehealth Forum \& Resource Centre ran webinars for health providers on telehealth consultations ... NZ Telehealth Forum \& Resource Centre provided information for both health providers and consumers on how to run telehealth consultations".

(UK):" Engaging healthcare professionals and the population regularly during the pandemic was essential in providing uniform trusted information. Communication directly with healthcare professionals is via a central web-based cascading alerting system for issuing health messages and other safety critical information and guidance. Communication with the population is through a government website".

(Brazil):" The Brazilian Telemedicine and Telehealth (ABTMS) and Health Informatics (SBIS) Societies have been promoting actions through online seminars and courses throughout the country, with emphasis on the Telemedicine University Network (RUTE) by means of the Special Interest Groups (SIG)".

(Jordan): "It has become clear that the vast majority of health care workers have 
never been exposed to such training or orientation. The need for training was clearly demonstrated as physicians and nurses were unfamiliar with the appropriate procedures for documentation, diagnosis and follow up of cases coming to them using telehealth services."

\section{Systems Framework for Telehealth Implementation to Support Critical Response}

The six themes from this paper highlight that telehealth delivery is a complex sociotechnical endeavour with technical, informatics, human resource, financial, and governance components. To provide a system framing for telehealth implementation in times of critical response such as during a global pandemic, we mapped our themes to the WHO Health System Framework [17]. Table 1 shows the mapping as a systems framework for telehealth implementation to support critical response. The systems framework provides guidance to assist with the conceptualization and implementation of telehealth to support critical response such as the COVID-19 pandemic.

While our framework uses the WHO Health Systems model, it extends the model by framing the system components for telehealth delivery. A systems perspective on telehealth provides insight that goes beyond clinical care aspects of telehealth such as supporting coordination of a broader health workforce as part of pandemic management and understanding the governance and financial implications of telehealth delivery.

\section{Discussion}

In this paper we described the international experiences in ten countries of implementing telehealth systems as part of managing the COVID-19 pandemic. We then use the international perspectives to develop a systems-based framework for telehealth implementation to support critical response. While our countries represented a diversity

Table 1 Systems framework for telehealth implementation to support critical response

\begin{tabular}{|c|c|}
\hline $\begin{array}{l}\text { WHO Health System Model } \\
\text { Component }\end{array}$ & Telehealth Configuration in Critical Response \\
\hline Service Delivery & $\begin{array}{l}\text { - Telehealth enabled virtual care delivery was provided for a significant proportion of the } \\
\text { population, leaving in-person care for those that needed it most. } \\
\text { - Health service delivery needs evolved over the course of the pandemic and functionalities } \\
\text { of telehealth tools needed to evolve too. }\end{array}$ \\
\hline Health workforce & $\begin{array}{l}\text { - Beyond formal healthcare workers, volunteers have played a major role in working side by } \\
\text { side with the governments and health systems to address the pandemic. } \\
\text { - Telehealth and other virtual care tools enabled coordination for caregivers and other } \\
\text { non-medical personnel to aid in care delivery. } \\
\text { - Telehealth went from being just a clinical tool to being a "care systems support tool" that } \\
\text { was instrumental in mobilising the broader health workforce. }\end{array}$ \\
\hline Information & $\begin{array}{l}\text { - System-wide networks of innovation including vendors and mobile apps have collectively } \\
\text { enabled effective communication and flow of information necessary for pandemic } \\
\text { management. } \\
\text { - Mobile tools and apps helped not only in dissemination of information to support care } \\
\text { delivery but also educational activities, which was critical given the rapid uptake of } \\
\text { telehealth in response to COVID-19. }\end{array}$ \\
\hline $\begin{array}{l}\text { Medical products, vaccines, } \\
\text { and technologies }\end{array}$ & $\begin{array}{l}\text { - Telehealth supported continuity of care for management of chronic conditions during } \\
\text { lock down conditions when face-to-face encounter was not possible. It also supported } \\
\text { peripheral tasks such as re-filling of prescription medications and provision of equipment } \\
\text { or other at home supports. } \\
\text { - The array of digital tools used ranged from telephone consults to formal and informal } \\
\text { telehealth tools. While variation in Telehealth usage was helpful in responding to } \\
\text { COVID-19, systems that evolve independent of formal medical informatics data standards } \\
\text { development may erode existing work on standards. }\end{array}$ \\
\hline Financing & $\begin{array}{l}\text { - Governments were not the only major funders of digital innovations, but international } \\
\text { donor agencies and the World Bank also played a major role in keeping the funding } \\
\text { streams flowing when businesses were "down". } \\
\text { - New fee codes or billing schedules may be needed for provider compensation of telehealth } \\
\text { delivery. It is essential that billing codes for virtual care remain in place after the pandemic. }\end{array}$ \\
\hline Leadership and governance & $\begin{array}{l}\text { - The World Health Organisation, the telemedicine societies, and the ministries of health in } \\
\text { the different countries played a major role in providing leadership and governance. } \\
\text { - Patients, caregivers, care providers, and other end users must be part of the planning and } \\
\text { implementation of telehealth tools. }\end{array}$ \\
\hline
\end{tabular}

of geographical, cultural, and socioeconomic contexts, as well as a variety of successes in responding to the COVID-19 pandemic, we still found many similarities related to telehealth use during the pandemic. All countries showed innovation to get telehealth rapidly implemented in response to the pandemic. We also saw a variation in the telehealth tools that were used with systems ranging from formal telehealth systems to tools such as WhatsApp being used as an informal telehealth system. Legal or regulatory issues were also experienced by all countries contributing to our paper with Governments worldwide showing flexibility in areas such as enabling remote consultations and developing flexible billing models. Government flexibility occurred despite the different health system delivery models (e.g., centralized or decentralized) across the countries in our paper. For example, in Canada healthcare is funded federally but implemented at the Provincial or Territory level but all Provinces and Territories developed new fee schedules to allow practitioners to bill for telehealth services. 
Public engagement was a particularly interesting theme that emerged out of our analysis. Patient and public engagement is necessary for any HIT initiative and many of our international perspectives talked about the role that volunteers played in responding to the pandemic such as delivering food or medication to people who needed it. Telehealth played a key role in coordinating volunteers and we cannot only think if it as a care delivery system. Telehealth can also be an enabler and coordinator of a broader health system response to a global pandemic.

There are several lessons learned from the global telehealth response to COVID-19. Globally we saw a wide variety of telehealth tools that were used in response to COVID- 19 . Early in the pandemic providers were using whatever tools where available, and while this agility was essential to the pivot to virtual care in response to the COVID-19 pandemic, it also increased the variation of telehealth tools that were used. While such diversity is not necessarily a bad thing, it does threaten to erode international standard development for telehealth delivery. Ad-hoc tools also can lead to privacy or security issues as they may not have been designed to meet privacy and security regulations.

Critical response that extends over a significant time such as the COVID-19 pandemic also presents emerging challenges. Initial response to COVID-19 was focused on stabilizing care delivery in the moment by coordinating fundamental care delivery needs such as access to basic medical services and procurement of medications for chronic disease management. As the pandemic progressed, telehealth needs evolved as people's medical needs become more complex and mental health issues became a significant consequence of social isolation and repeated lockdowns. More complex medical procedures such as cancer screening and other assessments or diagnoses that require physical touch are challenging to do virtually. Future research is needed to understand how to best utilize virtual care delivery $[5,28,29]$.

The COVID-19 pandemic has shown us that we can implement HIT such as telehealth faster than we normally do. This newfound agility must not be lost in a postCOVID-19 world. However, we do need to ensure that our quest for agile system design and implementation does not come at the cost of introducing unintended negative consequences into telehealth delivery. We cannot be so fixated on agile and rapid implementation that we ignore the need for interoperable standards and privacy and security aspects of informatics driven systems. Another challenge is that people that were able to access telehealth were often those that possessed appropriate technology and were more technically savvy. We need to ensure that telehealth delivery is done so it benefits all citizens and does not increase the gap between the haves and the have nots.

We are not here to solve telehealth implementation for COVID-19 but rather to make recommendations on how we can better enable telehealth capacity to support critical response such as during a global pandemic. The COVID-19 pandemic forced the world to become digitally connected and it is important that we do not lose the new digital innovations that were developed in response to COVID-19. We need to use COVID-19 as an opportunity to drive health system innovation for informatics tools such as Telehealth. Telehealth delivery is very much a systems-based endeavour and systems thinking can help us understand all the components that must be aligned in HIT implementation. The main contribution from this paper is a systems framework for telehealth implementation that provides guidance for the design and implementation of telehealth tools to support critical response.

One key limitation of our study is that the international perspectives are all from members of the IMIA Telehealth WG. As we are all experts in various aspects of HIT and Telehealth, as well as the broader discipline of health informatics, we bring a degree of bias to our analysis and the recommendations from this paper. Applying and studying our systems framework in different health systems will help to validate the framework.

\section{References}

1. Monaghesh E, Hajizadeh A. The role of telehealth during COVID-19 outbreak: a systematic review based on current evidence. BMC Public Health 2020;20:1193.

2. Fagherazzi G, Goetzinger $\mathrm{C}$, Rashid MA, Aguayo GA, Huiart L. Digital health strategies to fight COVID-19 worldwide: challenges, recommen- dations, and a call for papers. J Med Internet Res 2020;22(6):e19284.

3. Whitelaw S, Mamas MA, Topol E, Van Spall HGC Applications of digital technology in COVID-19 pandemic planning and response. Lancet Digi Health 2020 Aug;2(8):e435-e440.

4. Mort M, Finch T. Principles for telemedicine and telecare: The perspective of a citizens' panel. J Telemed Telecare 2005;11:66-8.

5. Reeves JJ, Hollandsworth HM, Torriani FJ, Taplitz $\mathrm{R}$, Abeles S, Tai-Seale M, et al. Rapid response to COVID-19: health informatics support for outbreak management in an academic health system. J Am Med Inform Assoc 2020;27(6):853-9.

6. Ash JS, Sittig DF, Dykstra RH, Guappone K, Carpenter JD, Seshadri V. Categorizing the unintended sociotechnical consequences of computerized provider order entry. Int J Med Inform 2007;76 Suppl 1:S21-7.

7. Wosik J, Fudim M, Cameron B, Gellad ZF, Cho A, Phinney D, et al. Telehealth transformation: COVID-19 and the rise of virtual care. J Am Med Inform Assoc 2020;27(6):957-62.

8. Thomas EE, Haydon HM, Mehrotra A, Caffery LJ, Snoswell CL, Banbury A, et al. Building on the momentum: Sustaining telehealth beyond COVID-19. J Telemed Telecare 2020;1357633X20960638.

9. Kuziemsky CE, Gogia SB, Househ M, Petersen C, Basu A. Balancing Health Information Exchange and Privacy Governance from a Patient-Centred Connected Health and Telehealth Perspective: A Contribution from the IMIA Organizational and Social Issues and Telehealth Working Groups. Yearb Med. Inform 2018; 27:48-54.

10. Kaplan B. Revisting Health Information Technology Ethical, Legal, and Social Issues and Evaluation: Telehealth/Telemedicine and COVID-19. Int J Med Inform 2020;143:104239.

11. Borycki EM, Kushniruk AW, Bellwood P, Brender J. Technology-induced errors. Methods Inf Med 2012;51(2):95-103.

12. Gogia SB, Maeder A, Mars M, Hartvigsen G, Basu A, Abbott P. Unintended consequences of tele health and their possible solutions: contribution of the IMIA working group on telehealth. Yearb Med Inform 2016 Nov 10;(1):41-6.

13. Harrison MI, Koppel R, Bar-Lev S. Unintended consequences of information technologies in health care - an interactive sociotechnical analysis. J Am Med Inform Assoc 2007;14:542-9.

14. Kuziemsky CE, Randell R, Borycki EM. Understanding Unintended Consequences and Health Information Technology: Contribution from the IMIA Organizational and Social Issues Working Group. Yearb Med Inform 2016 Nov 10;(1):53-60.

15. Mosnaim GS, Stempel H, Van Sickle D, Stempel DA. The Adoption and Implementation of Digital Health Care in the Post-COVID-19 Era. J Allergy Clin Immunol Pract 2020;8(8):2484-6.

16. Legido-Quigley H, Asgari N, Teo YY, Leung GM, Oshitani H, Fukuda K, Cook AR, et al. Are high-performing health systems resilient against the COVID-19 epidemic? Lancet 2020;395(10227):848-50.

17. De Savigny D, Adam T. Systems thinking for health systems strengthening. World Health 
Organization; 2009.

18. WHO. Health Systems Governance. Health systems governance [Internet]. 2020 [cited 2020 Dec 10]. Available from: https://www.who.int/westernpacific/health-topics/health-systems-governance

19. Adam T, de Savigny D. Systems thinking for strengthening health systems in LMICs: need for a paradigm shift. Health policy and planning 2012;27 Suppl 4:iv1-3.

20. Chang AY, Ogbuoji O, Atun R, Verguet S. Dynamic modeling approaches to characterize the functioning of health systems: A systematic review of the literature. Soc Sci Med 2017;194:160-7.

21. Champion C, Kuziemsky C, Affleck E, Alvarez GG. A systems approach for modeling health information complexity. Int J Inf Manage 2019;49:343-54.

22. Smith AC, Thomas E, Snoswell CL, Haydon H, Mehrotra A, Clemensen J, et al. Telehealth for global emergencies: Implications for coronavirus disease 2019 (COVID-19). J Telemed Telecare 2020;26:309-13.
23. Basu A. Data for Managing COVID-19 [Internet] 2020 [cited 2020 Dec 10]. Available from: https:// github.com/arinbasu/2020-12-07-imia_paper

24. Sacks E, Morrow M, Story WT, Shelley KD, Shanklink D, Rahimtoola M, et al. Beyond the building blocks: integrating community roles into health systems frameworks to achieve health for all. BMJ Glob Health 2019;3 (Supp13):e001384.

25. Kuziemsky CE, Hunter I, Gogia SB. Ethics in Telehealth: Comparison between Guidelines and Practice-based Experience-the Case for Learning Health Systems. Yearb Med Inform 2020 Aug;29(1):44-50.

26. Kulatunga GG, Hewapathirana R, Marasinghe R, Dissanayake VHW. A review of Telehealth practices in Sri Lanka in the context of the COVID-19 pandemic. Sri Lanka Journal of Bio-Medical Informatics 2020;11(1): 8-19.

27. Today C. App introduced to consult doctors. CeylonToday [Internet] 2020 [cited $2021 \mathrm{Feb}$ 17]. Available from: https://ceylontoday.lk/news/ app-introduced-to-consult-doctors.
28. Singh Bhandari S, Joseph SJ, Udayasankaran JG, Konthoujam B, Shoib S, Dutta S. Telepsychiatry: a feasible means to bridge the demand-supply gaps in mental health services during and after the COVID-19 pandemic: preliminary experiences from Sikkim state of India. Indian J Psychol Med 2020;42:500-2.

29. Haranath SP, Udayasankaran JG. Tele-intensive care unit networks: A viable means for augmenting critical care capacity in India for the COVID pandemic and beyond. Apollo Medicine 2020;17:209.

\section{Correspondence to:}

Arindam Basu

School of Health Sciences

University of Canterbury

405F, Manawa

176 St Asaph Street

Christchurch 8011, New Zealand

Tel: +64033693509

E-mail: arindam.basu@canterbury.ac.nz 